\title{
Determinants of Stroke Mortality through Survival Models: The Case of Mettu Karl Referral Hospital, Mettu, Ethiopia
}

\author{
Dereje Gebeyehu Ababu (iD) and Azmeraw Misganaw Getahun \\ Department of Statistics, Faculty of Natural \& Computational Science, Mettu University, Ethiopia \\ Correspondence should be addressed to Dereje Gebeyehu Ababu; derejegebe@gmail.com
}

Received 16 March 2021; Accepted 25 January 2022; Published 11 February 2022

Academic Editor: Augusto Fusco

Copyright (C) 2022 Dereje Gebeyehu Ababu and Azmeraw Misganaw Getahun. This is an open access article distributed under the Creative Commons Attribution License, which permits unrestricted use, distribution, and reproduction in any medium, provided the original work is properly cited.

\begin{abstract}
Introduction. Every year worldwide, between five to six million deaths are associated with stroke; on average, one stroke-related death occurs every four minutes. In Ethiopia, stroke is a frequent cause of mortality and morbidity from noncommunicable diseases. Therefore, this study was aimed at determining factors associated to stroke mortality through survival models in Mettu Karl Referral Hospital. Methods. This study was conducted from September 1, 2014, to April 1, 2017, and encompassed 202 stroke patients at Mettu Karl Referral Hospital. The Cox semiparametric regression was used for analyzing survival analysis of stroke patients using $\mathrm{R}$ software. Results. A total of 202 stroke patients were included in the study, and among those patients, $72.8 \%$ and $27.2 \%$ were censored and died, respectively. According to the result of Cox semiparametric regression model, sex of patients, hypertension, baseline complication, and stroke type had significant effect on survival of the stroke patient at 5\% significance level. Conclusion. The results from Cox semiparametric regression model indicated that sex of patients, hypertension, baseline complication, and stroke type were major factors related to the survival time of stroke patients. The researcher recommends that the people should be aware on the burden of those risk factors and well informed about the disease.
\end{abstract}

\section{Introduction}

Globally, stroke is the second leading cause of death above the age of 60 years and about 16 million new cases of stroke and 62 million stroke survivors were estimated in 2005, with deaths from stroke accounting for $9.7 \%$ of all global deaths, and this is expected to increase to over 23 million new stroke cases and 7.8 million stroke deaths by 2030 in the absence of significant global public health response [1].

The study conducted in south western Saudi Arabia showed that prestroke smoking, prestroke hypertension, poststroke disturbed consciousness poor mobility, and developing pulmonary embolism as significant predictors of in-hospital stroke mortality [2].

In Africa, the impact of stroke is increasing due to a rising prevalence of hypertension associated with a very poor level of awareness and control. African countries are undergoing an epidemiological transition driven by socio- demographic and lifestyle changes [3]. The burden of noncommunicable diseases (NCD), including cardiovascular risk factors, is increasing. Consequently, the incidence of stroke, a cardinal complication of cardiovascular risk factors, appears to be rising in Africa [4].

In Ethiopia, hypertension was the most important risk factor identified in 1990. However, during that time, computed tomography (CT) scan was not available to differentiate stroke subtypes and the modern approach in treating patients with strokes requires early CT scan as it is imperative to diagnose whether the stroke is ischemic or hemorrhagic [5].

Although Ethiopia is progressing towards universal health coverage, the country faces the double burden of both communicable and noncommunicable diseases [6]. According to the report of WHO in 2017, stroke deaths in Ethiopia reached $6.23 \%$. Moreover, the age-adjusted death rate of stroke in Ethiopia was 89.82 per 100,000 of the population. 
TABLE 1: Baseline covariate characteristics with their time-to-death status.

\begin{tabular}{lcccccc}
\hline Variables & Categories & Frequency & Death (\%) & Censored (\%) & Mean (days) & {$[95 \% \mathrm{CI}]$} \\
\hline \multirow{2}{*}{ Gender } & Male & $120(59.4 \%)$ & $34(55.7 \%)$ & $86(61 \%)$ & 9.179131 & {$[8.04889,10.3094]$} \\
& Female & $82(40.6 \%)$ & $27(44.3 \%)$ & $55(39 \%)$ & 5.150107 & {$[4.22813,6.07208]$} \\
Blood pressure & Yes & $123(60.9 \%)$ & $44(72.13 \%)$ & $77(54.6 \%)$ & 5.761887 & {$[5.15259,6.37119]$} \\
& No & $79(39.1 \%)$ & $17(27.87 \%)$ & $64(45.4 \%)$ & 9.764186 & {$[8.30564,11.2227]$} \\
Cardiac disease & Yes & $26(12.9 \%)$ & $11(20 \%)$ & $15(10.2 \%)$ & 8.069148 & {$[5.14086,10.9974]$} \\
& No & $176(87.1 \%)$ & $44(80 \%)$ & $132(89.8 \%)$ & 7.770081 & {$[6.85184,8.68832]$} \\
Diabetes mellitus & Yes & $15(7.4 \%)$ & $3(5.45 \%)$ & $12(8.2 \%)$ & 6.289487 & {$[4.67067,7.9083]$} \\
& No & $187(92 \%)$ & $52(94.54 \%)$ & $135(91.8 \%)$ & 7.918552 & {$[6.98651,8.8506]$} \\
Stroke type & Hemorrhagic & $70(34.7 \%)$ & $29(52.73 \%)$ & $41(27.9 \%)$ & 6.137696 & {$[5.17646,7.09893]$} \\
& Ischemic & $117(57.9 \%)$ & $23(41.82 \%)$ & $94(63.9 \%)$ & 8.719683 & {$[7.5657,9.87366]$} \\
\multirow{2}{*}{ Baseline complication } & Both & $15(7.4 \%)$ & $3(5.45 \%)$ & $12(8.2 \%)$ & 4.584416 & {$[3.25288,5.91595]$} \\
& Yes & $115(56.9 \%)$ & $37(67.3 \%)$ & $78(53 \%)$ & 5.391817 & {$[4.83329,5.95035]$} \\
& No & $87(43.1 \%)$ & $18(32.7 \%)$ & $69(47 \%)$ & 9.92229 & {$[8.54041,11.3042]$} \\
\hline
\end{tabular}

Kaplan-Meier survival estimate

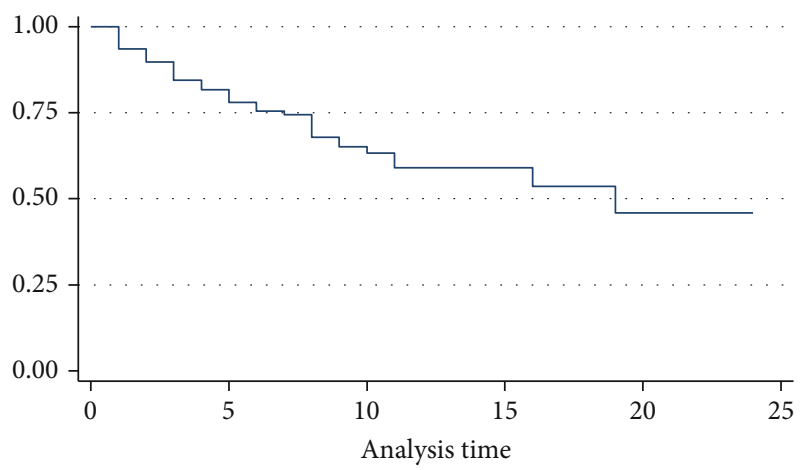

FIgURE 1: Overall Kaplan-Meier survivor function.

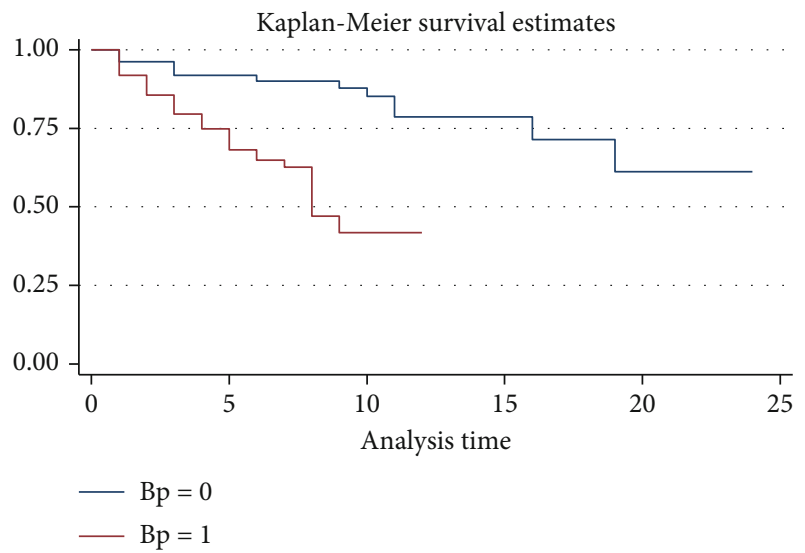

Figure 2: K-M plot survival of time-to-death by hypertension of stroke patients.

Earlier reports showed that the future trend of stroke in SubSahara Africa will increase over the coming years owing to poor healthcare and poor neurologic interventions [7].

Therefore, chronic diseases become major global public health problems, mainly, in developing countries. However, there is limited evidence on the magnitude and factors of
TABLE 2: Log rank tests of each covariate.

\begin{tabular}{lccc}
\hline Variable & \multicolumn{3}{c}{ Log rank test } \\
& Chi.sq & Df & $P$ value \\
\hline Gender & 27.4 & 1 & $<0.001$ \\
Age & 100 & 52 & $<0.001$ \\
Cardiac disease & 0 & 1 & 0.988 \\
Diabetes mellitus & 0.9 & 1 & 0.341 \\
Blood pressure (hypertension) & 22.3 & 1 & $<0.001$ \\
Drug type & 9.4 & 6 & 0.152 \\
Baseline complication & 31.4 & 1 & $<0.001$ \\
Stroke type & 8.1 & 2 & 0.150 \\
\hline
\end{tabular}

stroke in Ethiopia using survival models, particularly, in Mettu Karl Referral Hospital, which is the aim of this study. Findings of this study were important evidence to the studied hospital and Oromia Regional Health Bureau to know the burden of stroke and determinant factors and plan appropriate preventive methods against stroke.

\section{Methodology}

2.1. Study Area. This study was conducted in Mettu Karl Referral Hospital. This study was conducted from the $1^{\text {st }}$ of September 2014 to the $1^{\text {st }}$ of April 2017 in Mettu Karl Hospital.

2.2. Study Design and Population. Retrospective data were gathered from a total number of 202 stroke disease patients diagnosed with stroke case patients in the medical and surgical wards from the $1^{\text {st }}$ of September 2014 to the $1^{\text {st }}$ of April 2017 in MCRH.

2.3. Variable in the Study. The dependent (outcome) variable in this study was the survival time measured (in days) from the start date of stroke treatment until the date of the patient's death or censor. The following are covariate variables in the study: sex, age, hypertension, cardiac disease, 
TABLE 3: Summary univariable (single covariate) Cox PH analysis.

\begin{tabular}{|c|c|c|c|c|c|c|c|}
\hline Covariate & Category & Coef $(\beta)$ & HR & se $(\beta)$ & Wald & $\operatorname{Pr}(>|z|)$ & $95 \% \mathrm{CI}$ \\
\hline Age & & 0.011662 & 1.011731 & 0.004634 & 6.33 & 0.012 & {$[1.003,1.0210]$} \\
\hline \multirow{2}{*}{ Gender } & 0 female & Ref & & & & & \\
\hline & 1 male & -0.8860 & 0.4123 & 0.1845 & 23.05 & $<0.001$ & {$[0.2872,0.5920]$} \\
\hline \multirow{2}{*}{ Cardiac disease } & 0 no & Ref & & & & & \\
\hline & 1 yes & -0.003844 & 0.996164 & 0.273690 & -0.014 & 0.989 & {$[0.5826,1.7030]$} \\
\hline \multirow{2}{*}{ Diabetes mellitus } & 0 no & Ref & & & & & \\
\hline & 1 yes & 0.2712 & 1.3116 & 0.3046 & 0.79 & 0.373 & {$[0.7219,2.3830]$} \\
\hline \multirow{2}{*}{ Hypertension } & 0 no & Ref & & & & & \\
\hline & 1 yes & 0.9365 & 2.5510 & 0.1986 & 22.24 & $<0.001$ & {$[1.7290,3.7650]$} \\
\hline \multirow{3}{*}{ Stroke type } & 0 (hemorrhagic) & Ref & & & & & \\
\hline & 1 (ischemic) & -0.4857 & 0.6152 & 0.1976 & -2.458 & 0.014 & {$[0.4177,0.9062]$} \\
\hline & 2 (both type) & 0.5496 & 1.7325 & 0.3303 & 13.44 & 0.096 & {$[0.9068,3.3101]$} \\
\hline \multirow{2}{*}{ Baseline complication } & 0 no & Ref & & & & & \\
\hline & 1 yes & 1.1220 & 3.0710 & 0.2056 & 29.78 & $<0.001$ & {$[2.052,4.5950]$} \\
\hline
\end{tabular}

TABle 4: Summary multivariable Cox PH analysis to the stroke data set from the MCRH.

\begin{tabular}{|c|c|c|c|c|c|c|c|}
\hline Covariates & Category & Coef $(\beta)$ & Haz. ratio & Std. Err. $(\beta)$ & Wald & $P>|z|$ & [95\% Conf. Interval HR] \\
\hline Age & & 0.0051853 & 1.0051988 & 0.0052631 & 0.985 & 0.325 & {$[0.9949,1.0156]$} \\
\hline \multirow{2}{*}{ Gender } & 0 female & Ref & & & & & \\
\hline & 1 male & -0.7226692 & 0.4854547 & 0.1937196 & -3.730 & $<0.001$ & {$[0.3321,0.7096]$} \\
\hline \multirow{2}{*}{$\begin{array}{l}\text { Blood pressure } \\
\text { (hypertension) }\end{array}$} & 0 no & Ref & & & & & \\
\hline & 1 yes & 0.6713666 & 1.9569097 & 0.2145444 & 3.129 & 0.002 & {$[1.2851,2.9798]$} \\
\hline \multirow{2}{*}{ Baseline complication } & $0($ no) & Ref & & & & & \\
\hline & 1 (yes) & 0.5218596 & 1.6851584 & 0.2236382 & 2.333 & 0.020 & {$[1.0871,2.6122]$} \\
\hline \multirow{3}{*}{ Stroke type } & 0 (hemo) & Ref & & . & & & . \\
\hline & 1 (ische) & -0.2105377 & 0.8101485 & 0.2094069 & -1.005 & 0.315 & {$[0.5374,1.2213]$} \\
\hline & 2(both) & 0.6232302 & 1.8649425 & 0.3409207 & 1.828 & 0.068 & {$[0.9560,3.6380]$} \\
\hline
\end{tabular}

diabetes mellitus, stroke type, baseline complication, and drug type.

2.4. Method of Data Analysis. Survival analysis is a collection of statistical procedures for data analysis for which the outcome variable of interest is time until an event occurs. An initial step in the analysis of a set of survival data is to present numerical or graphical summaries of the survival times in a particular group. In summarizing survival data, the two common functions of applied are the survivor function and the hazard function [6].

The Kaplan-Meier estimator is a nonparametric statistic used to estimate the survival function from lifetime data.

The Kaplan-Meier estimator of the survivorship function (or survival probability) $S(t)=P(T \geq t)$ is defined as

$$
\widehat{S}(t)=\prod_{t_{i} \leq t} \frac{n_{i}-d_{i}}{n_{i}}=\prod_{t_{i} \leq t} 1-\frac{d_{i}}{n_{i}},
$$

where $t_{1}, t_{2}, \cdots, t_{n}$ a set of survival time of $n$ independent observations and $t_{(1)} \leq t_{(2)} \leq \cdots \leq t_{(m)}, m \leq n$ is the survival time of the $m$ distinct ordered death times; $d_{i}$ is the number of individuals who failed (died) at time $t_{i}$; and $n_{i}$ is the number of individuals who are at risk of dying at time $t_{i}$.

One of the most popular types of regression models used in survival analysis is the Cox proportional hazard model [8]. The authors proposed a semiparametric model for the hazard function that allows the addition of covariates, while keeping the baseline hazards unspecified, and only positive values with this parameterization the Cox hazard function are given by

$$
h(t, x)=h_{o}(t) \exp (\beta x)
$$

where $h_{0}(t)$ is the baseline hazard function and is called the baseline hazard function; $x=\left(x_{1}, x_{2}, \cdots, x_{p}\right)^{\prime}$ is the values of the vector of explanatory variables; and $\beta^{\prime}=\left(\beta_{1}, \beta_{2}, \cdots, \beta_{p}\right)$ is a vector of regression coefficients. The main assumption of the Cox proportional hazard model is proportional hazards that means that the hazard function of one individual is proportional to the hazard function of the other individual; i.e., the hazard ratio is constant over time. 


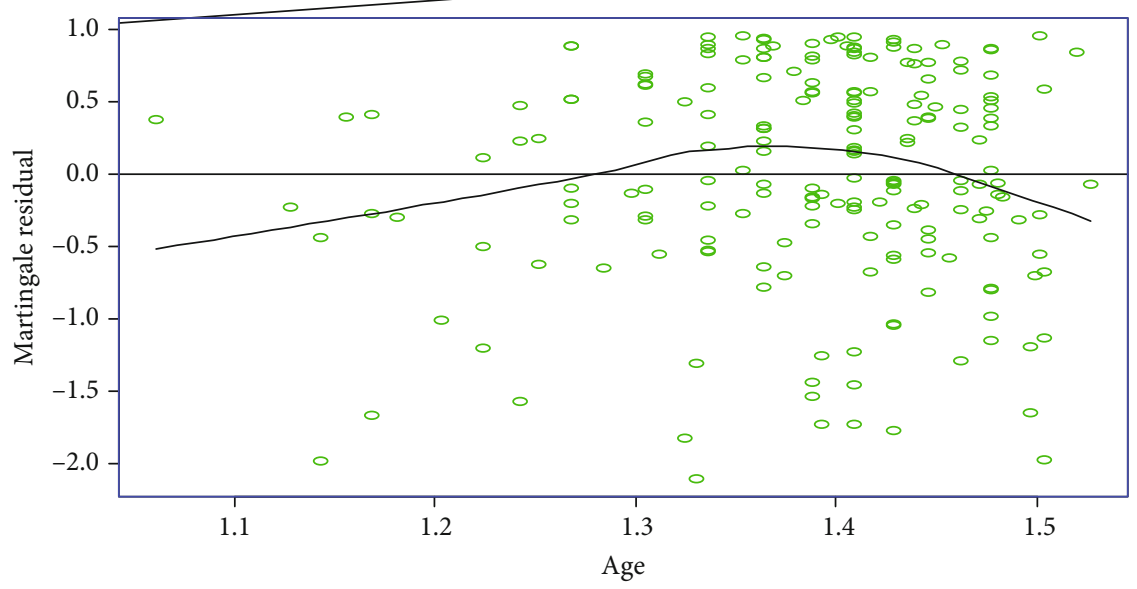

FIgURE 3: Plots of the martingale residuals against the covariate age.

\section{Result and Discussion}

3.1. Descriptive Summaries. A total of 295 stroke patients were treated in the hospital during the study period from the $1^{\text {st }}$ of September 2014 to the $1^{\text {st }}$ of April 2017. Of total population, this study included 202 stroke patients for whom data for variables of interest are complete. Of all 202 stroke patients, $147(72.8 \%)$ were censored or did not experience the event and $55(27.2 \%)$ died. Average time duration for all patients was 6.05 with a standard deviation of 4.698 , and the median and mean survival time of age were 6 and 7.168 days, respectively. The mean and median age of stroke patient were 62.56 and 65 days, respectively. The mean and median survival time from stroke were found to be 15.596 and 19 days, respectively. The mean and median survival time from stroke were found to be 15.59619 days, respectively. The mean survival time of male and female was 9.1 days and 5.1 days, respectively. The minimum and maximum survival times observed in the data were 1 day and 24 days, respectively. The median survival time of female and male was 3 days and 6 days, respectively. Patients with hypertension stayed for 4 days of which $72.13 \%$ were death. However, patients with no hypertension stayed for 9 days on average of which $27.87 \%$ were death. Patients with baseline complication stayed for 4 days on average of which $67.3 \%$ were death (Table 1 ).

3.2. The Kaplan-Meier Estimate. The estimate for overall Kaplan-Meier survivor function depicted that a relatively large number of the deaths occurred at the earlier days of antistroke treatment, and the same graph showed the decrement over a follow-up period (Figure 1).

Kaplan-Meier estimates represented by the survival curves for without hypertension diseases are above those of the patients' complications with hypertension. This implied that the patients without hypertension have more chances of survival than those with hypertension (Figure 2).

3.3. Log-Rank Tests of Each Covariate. The log-rank test indicates that statistically there is a significant difference of survival experience among groups of gender, age, blood pressure (hypertension), and baseline complication. On the other hand, there are statistically no significant differences in survival/death experience among groups of the categorical covariates cardiac disease, diabetes mellitus, stroke type, and drug type. Accordingly, the mean survival time of male patient to death had been 9.1 days greater than that of female patients (5.1 days with $95 \%$ CI $[8.04889,10.3094]$ ) (Table 2).

3.4. Univariable Analysis of Cox PH Regression Model. From the outputs in univariable analysis, we can observe that the covariate age of stroke patient $(\mathrm{HR}=1.011731, P$ value $=$ $0.0119)$, gender of patient $(\mathrm{HR}=0.4123, P$ value $=1.58 e-$ 06), hypertension $(\mathrm{HR}=2.5510, P$ value $=2.40 e-06)$, stroke type $(\mathrm{HR}=0.6152,1.7325 ; P$ value $=0.014,0.0961)$, and drug type and baseline complication $(\mathrm{HR}=3.0710, P$ value $=4.84 e-08)$ are significant and hypertension, baseline complication, gender, and stroke type are highly significant in the univariable analysis. However, diabetes mellitus and cardiac are not significant factors for the death time at 5\% level of significance (Table 3). This result is consistent with a previous study $[3,9]$ that age of patients was a significant factor in affecting the survival of stroke patients.

3.5. Multivariable Analysis of Cox PH Regression Model. Covariates which become insignificant in the multivariable analysis were removed from the model by using a stepwise elimination technique. Accordingly, cardiac disease and diabetes mellitus were excluded. In order to decide whether or not a variable is significant, the $P$ value associated with each parameter has been estimated and variables that have $P$ value less than 0.05 cut-point or $5 \%$ significance level are considered important variables and, hence, are included in the final model. Accordingly, sex, hypertension, baseline complication, and stroke type had significant effect on the survival of the stroke patient (Table 4). This result is supported by research conducted in China [5] in that hypertension was a significant factor in affecting the survival of stroke patients. The result is also consistent with that of a previous 
TABLE 5: Schoenfeld residual for each covariate.

\begin{tabular}{|c|c|c|c|c|}
\hline Covariates & Categories & Rho & $\mathrm{Chi}^{2}$ & Prob $>$ chi $^{2}$ \\
\hline Age & & 0.0462 & 0.2586 & 0.611 \\
\hline \multirow{2}{*}{ Gender } & Female ref & & & \\
\hline & Male & 0.0304 & 0.1364 & 0.712 \\
\hline \multirow{2}{*}{ Hypertension } & No ref & & & \\
\hline & Yes & 0.1115 & 2.3567 & 0.125 \\
\hline \multirow{2}{*}{ Baseline complication } & No ref & & & \\
\hline & Yes & 0.0625 & 0.8123 & 0.367 \\
\hline \multirow{6}{*}{ Drug type } & Nondrug (ref) & & & \\
\hline & Drug type 1 & -0.0974 & 1.3874 & 0.239 \\
\hline & Drug type 2 & -0.0573 & 0.4887 & 0.485 \\
\hline & Drug type 3 & -0.0111 & 0.0177 & 0.894 \\
\hline & Drug type 4 & -0.0374 & 0.1989 & 0.656 \\
\hline & Drug type 5 & -0.0445 & 0.2939 & 0.588 \\
\hline \multirow{3}{*}{ Stroke type } & 0 ref & & & \\
\hline & Stroke type 1 & -0.0229 & 0.0874 & 0.768 \\
\hline & Stroke type 2 & 0.0360 & 0.1898 & 0.663 \\
\hline Global test & & & 8.4942 & 0.668 \\
\hline
\end{tabular}

TABLE 6: The likelihood ratio and significance of the $\mathrm{PH}$ model.

\begin{tabular}{lccccc}
\hline Covariate & Coef $(\beta)$ & HR & se $(\beta)$ & Wald & $P$ value \\
\hline Factor (gender) 1 & -0.712 & 0.491 & 0.193 & -3.69 & $<0.001$ \\
Factor (BP) 1 & 0.654 & 1.923 & 0.208 & 3.15 & 0.002 \\
Factor (baseline) 1 & 0.666 & 1.947 & 0.218 & 3.06 & 0.002 \\
\hline
\end{tabular}

Likelihood ratio test $=49.8$ on $3 \mathrm{DF} ; P=8.87 e-11 ; n=202$; number of events $=147$.

study [10] that sex of patients was a significant factor in affecting the survival of stroke patients. The finding of the study is also consistent with that of a previous study [9] that stroke mortality increased with age of patients.

3.6. Checking for the Linearity of Continuous Covariates in the Model. For the age covariate, the plots show systematic patterns or trends and the resulting smoothed plots are not a straight line. Therefore, the plots of martingale residual confirm that age of a patient has no linear relationship with the survival time (Figure 3).

3.7. Checking of Proportional Hazard Assumption. The test of correlation (rho) is insignificant that indicates proportional hazards assumption is fulfilled. Variables including age, gender of patients, hypertension (blood pressure), baseline complication, drug type, and stroke type fulfilled the assumption because all the $P$ values are greater than 0.05 . In Schoenfeld residual, if the $P$ value is greater than 0.05 it indicates that the Cox proportional hazard assumptions are fulfilled (Table 5).

3.7.1. Diagnosis of the Model. In the likelihood ratio and significance of the final Cox $\mathrm{PH}$ model from the likelihood ratio test, it can be seen that the $\mathrm{PH}$ model is significant since $P$ value is less than $5 \%$ (Table 6).

\section{Conclusions and Recommendation}

The objective of the study was to identify significant risk factors that affect survival of stroke patients who have been under follow-up at Mettu Karl Referral Hospital. For determining the risk factors for the survival time of stroke patients, a total of 202 patients were included in the study out of which $40.6 \%$ were females and $59.4 \%$ were males. Among those patients, $27.2 \%$ died and the rest were censored.

The Cox regression analysis showed that the major factors that affect the survival of stroke patients are hypertension, gender of the patients, and baseline complication which were strongly related to mortality, and based on the hospital outcome, the most common causes of death were hypertension and base line complication. However, there were variables that were significant at univariable stage of analysis but not at multivariate analysis stage. These were stroke type, drug type, and age of patients. Moreover, variables that were significant neither at univariable nor at multivariable analyses were gender, baseline complication, and hypertension. The result of this study also indicated that survival probability of a patient is not statistically different among groups classified by cardiac disease, diabetes mellitus, stroke type, and drug type.

The researchers recommend that the people should be aware on the burden of those risk factors and well informed about the disease. Finally, the researchers propose the concerned body special health management to improve the health of the society based on the result of the study.

\section{Limitations}

This research paper was limited to time from the date of the first diagnosis until the occurrence of the death or end of the 
study period for 202 stroke patients in case of Karl Hospital, Ilu Ababor Zone.

\section{Abbreviations}

AIC: Akaike's Information Criterion

AIDS: Acquired immune deficiency syndrome

BIC: Bayesian Information Criterion

CT: Computed tomography

HIV: Human immunodeficiency virus

NCD: Noncommunicable diseases

WHO: World Health Organization.

\section{Data Availability}

The data used to support the findings of this study are available from the corresponding author upon request.

\section{Ethical Approval}

This study was reviewed and approved by Mettu University Research Ethical Committee.

\section{Consent}

As the study was based on retrospective cohort study, informed consent was not needed.

\section{Conflicts of Interest}

The authors declare that there is no competing interest.

\section{Acknowledgments}

This work is licensed under a CC BY 4.0 License. Read Full License on 5 April 2021 based on the following link: https:// www.researchsquare.com/article/rs-366194/v1

\section{References}

[1] D. O. Abegunde, C. D. Mathers, T. Adam, M. Ortegon, and K. Strong, "The burden and costs of chronic diseases in lowincome and middle-income countries," Lancet, vol. 370, no. 9603, pp. 1929-1938, 2007, PMID: 18063029.

[2] A. A. Alhazzani, A. A. Mahfouz, A. Y. Abolyazid et al., "In hospital stroke mortality: rates and determinants in southwestern Saudi Arabia," International Journal of Environmental Research and Public Health, vol. 15, no. 5, p. 927, 2018.

[3] E. S. Donkor, M. O. Owolabi, P. Bampoh, T. Aspelund, and V. Gudnason, "Community awareness of stroke in Accra Ghana," BMC Public Health, vol. 14, no. 1, p. 196, 2014.

[4] A. Moran, M. Forouzanfar, U. Sampson, S. Chugh, V. Feigin, and G. Mensah, "The epidemiology of cardiovascular diseases in sub-Saharan Africa: the Global Burden of Diseases, Injuries and Risk Factors 2010 Study," Progress in Cardiovascular Diseases, vol. 56, no. 3, pp. 234-239, 2013.

[5] S. Yusuf, S. Reddy, S. Ounpuu, and S. Anand, "Global burden of cardiovascular diseases," Circulation, vol. 104, no. 23, pp. 2855-2864, 2001.
[6] A. Misganaw, T. N. Haregu, K. Deribe et al., "National mortality burden due to communicable, non-communicable, and other diseases in Ethiopia, 1990-2015: findings from the Global Burden of Disease Study 2015," Population Health Metrics, vol. 15, no. 1, p. 29, 2017.

[7] R. BeLue, T. A. Okoror, J. Iwelunmor et al., "An overview of cardiovascular risk factor burden in sub-Saharan African countries: a socio-cultural perspective," Globalization and Health, vol. 5, no. 1, p. 10, 2009.

[8] D. R. Cox and D. Oakes, Analysis of Survival Data, Chapman and Hall London Google Scholar, Boca Raton, 1st edition, 1984.

[9] Y. Sun, S. H. Lee, B. H. Heng, and V. S. Chin, "5-year survival and rehospitalization due to stroke recurrence among patients with hemorrhagic or ischemic strokes in Singapore," BMC Neurology, vol. 13, no. 1, p. 133, 2013.

[10] D. W. Hosmer, S. Lemeshow, and X. Rodney, Applied Logistic Regression, John Wiley \& Sons, Inc., New York, 2nd edition, 2000. 